\title{
Flexible Zielführung von Ladungsträgern in Produktion und Materialflusslogistik durch vollständig in den Materialfluss integrierte Flurförderzeuge
}

\author{
M. SC. DiPL.-ING. (FH) ANDREAS JUNGK \\ PROF. DR.-ING. LUDGER OVERMEYER \\ INSTITUT FÜR TRANSPORT- UND AUTOMATISIERUNGSTECHNIK, UNIVERSITÄT HANNOVER
}

Zusammenfassung

Die Fortschritte im Bereich der Kommunikations- und Informationstechnologien und eine globalisierte Wirtschaft stellen immer neue Anforderungen an die Logistikprozesse. Die Anpassung an diese Anforderungen wird in Zukunft vermehrt durch den Einsatz von dezentraler Informationstechnologie erfolgen. Die RFID-Technologie lässt neue Systemarchitekturen zu, bei denen kleine Subsysteme zu einem robusten und flexiblen Gesamtsystem verbunden werden. Als zentrale Schnittstelle soll das Flurförderzeug eingesetzt werden. Dies bedeutet zum einen die Kommunikation mit den übergeordneten DV-Systemen, auf der anderen Seite mit den Waren, Ladungsträgern, Übergabestationen und Arbeitsstationen. Verschiedene Ansätze aus dem RFID-Umfeld werden derzeit von FFZ-Herstellern verfolgt. Im Rahmen des BMBF-geförderten Forschungsvorhaben „IdentProLog“ wird das Flurförderzeug als Schlüsselelement im Logistikprozess dienen. Zur durchgängigen Steuerung des Materialflusses in einem Produktionsbetrieb wird ein Zielführungssystem entwickelt. Dabei realisiert das FFZ die Kommunikation zu den Leit-, Führungs- und Managementsystemen der EDV mittels RFIDTechnik und WLAN.

\section{Abstract}

The advances in communication and information technologies and a worldwide economy require new logistics processes. To fit these requirements more and more decentralized information technology will take place.The RFID technology permits new architectures. Small subsystems are connected to a durable and flexible system. The forklifter is also the central interface. On the one hand for communication with the superordinate data processing systems, on the other side with the goods, charge carriers, transfer stations and work stations. Currently the manufacters of forklifters have started different research activities. In the context of the BMBF promoted project IdentProLog the industrial trucks will serve as key element during the logistics process. For the constant controlling of the flow of material in a production enterprise a new guidance system is developed. The fork lifter realizes communication to the direction, guidance and management systems of the EDP by using RFID technology and WLAN.

\section{Einleitung}

Neue Technologien führen in einer globalisierten Wirtschaft dazu, dass sich nicht nur multinational agierende Grosskonzerne, sondern auch zunehmend kleine und mittelständische Unternehmen im internationalen Wettbewerb behaupten müssen. Damit steigen auch die Anforderungen an die innerbetrieblichen Materialflussund Warenumschlagsprozesse, also an die Intralogistik.

Insbesondere das Internet führt dazu, dass sich Angebote im internationalen Vergleich behaupten müssen. Hinzu kommen immer höhere Anforderungen hinsichtlich Liefertreue und -zeiten sowie der Dokumentierbarkeit des Logistikprozesses.

Kürzere Laufzeiten der Produktionsprozesse führen dazu, dass auch der Logistikprozess ständig angepasst werden muss. Statt starrer Anlagen werden sich also eher flexible Systeme durchsetzen, statt Großanlagen eher modularisierte, skalierbare Lösungen. 


\section{Anforderungen an die Intralogistik}

In diesem Kontext müssen die Anforderungen an eine zukünftige Intralogistik formuliert werden. Als wesentlich sind hierbei Kostenreduktionen und eine optimale Ausnutzung des Faktors Zeit zu nennen. Um dies zu erreichen, müssen Durchlaufzeit und Umlaufzeiten reduziert werden.

Um sich schnell an neue Prozesse anpassen zu können, muss die Intralogistik flexibler werden. Da das Logistiksystem eine Schlüsselfunktion in der Fertigung einnimmt, können Ausfallzeiten nicht toleriert werden. Ziel ist somit auch eine deutliche Steigerung der Robustheit und der Verfügbarkeit, also eine höhere Zuverlässigkeit.

Um Logistikprozesse optimieren zu können, ist eine hohe Transparenz des Prozesses notwendig. Auch aus rechtlichen Gründen muss daher die Überwachbarkeit und die Nachverfolgbarkeit verbessert werden.

\section{RFID-Technik als technologischer Enabler}

Die Radio Frequenz Identifikation (RFID) stellt ein Verfahren dar, das es erlaubt, Personen und Objekte eindeutig, schnell und berührungslos zu identifizieren. Das Potenzial der RFID erstreckt sich dabei über einfache Identifikationsaufgaben und bietet die Möglichkeit, Daten lokal zu speichern, sensorisch zu erfassen und am Objekt zu verarbeiten bzw. Steuerungsentscheidungen zu treffen. RFID-Systeme bestehen prinzipiell aus Transpondern, dem Schreib-/Lesegerät, der Antenne und Auswerteeinheit. Das Datenmedium der RFID sind Transponder, die je nach Ausführungs- und Verpackungsform auch als Smart Label, Tag oder elektronisches Etikett bekannt sind. Die Schreib-/Lesegeräte verfügen über eine Schnittstelle zu übergeordneten Systemen (Auswerteeinheit). In dieser werden alle von Schreib-/Lesegeräten erfassten Daten verarbeitet und sofern Daten auf einem Transponder gespeichert werden sollen, zu diesem zurück übertragen. Eine Differenzierung der verschiedenen RFID-Systeme ist nach unterschiedlichen Kriterien möglich - z. B. nach der Betriebsfrequenz, der Schreib-/Lesereichweite, der Speichergröße, der Funktionalität, der Energieversorgung oder des Kommunikationsprinzips. [Finkenzeller2002, Overmeyer2004b].

Die RFID-Techik bringt hier den Vorteil mit sich, zum einen größere Datenmengen als beim Barcode zu speichern, zum anderen während des Prozesses Daten auf dem Transponder zu speichern.

\section{Neue Möglichkeiten und Visionen}

Um der Forderung nach Flexibilität gerecht zu werden, ist es notwendig, an jeder Position, an der eine Warenübergabe möglich ist, Gates zu errichten. Die Lösung kann nur sein, mobile Gates einzusetzen, die immer an der benötigten Stelle installiert sind. Zweckmäßig ist es, das Flurförderzeug als mobiles Gate zu nutzen, da dieses den Materialfluss überall dort begleitet, wo Flexibilität gefordert ist.

Die technologischen Möglichkeiten erlauben es, einen neuen Logistikprozess zu gestalten, in dem das Flurförderzeug das zentrale Koppelelement im Material- und im Informationsfluss bildet.

Die Flurförderzeuge werden hierzu mit RFID- und WLAN-Technik ausgestattet. (Abb. 1). 


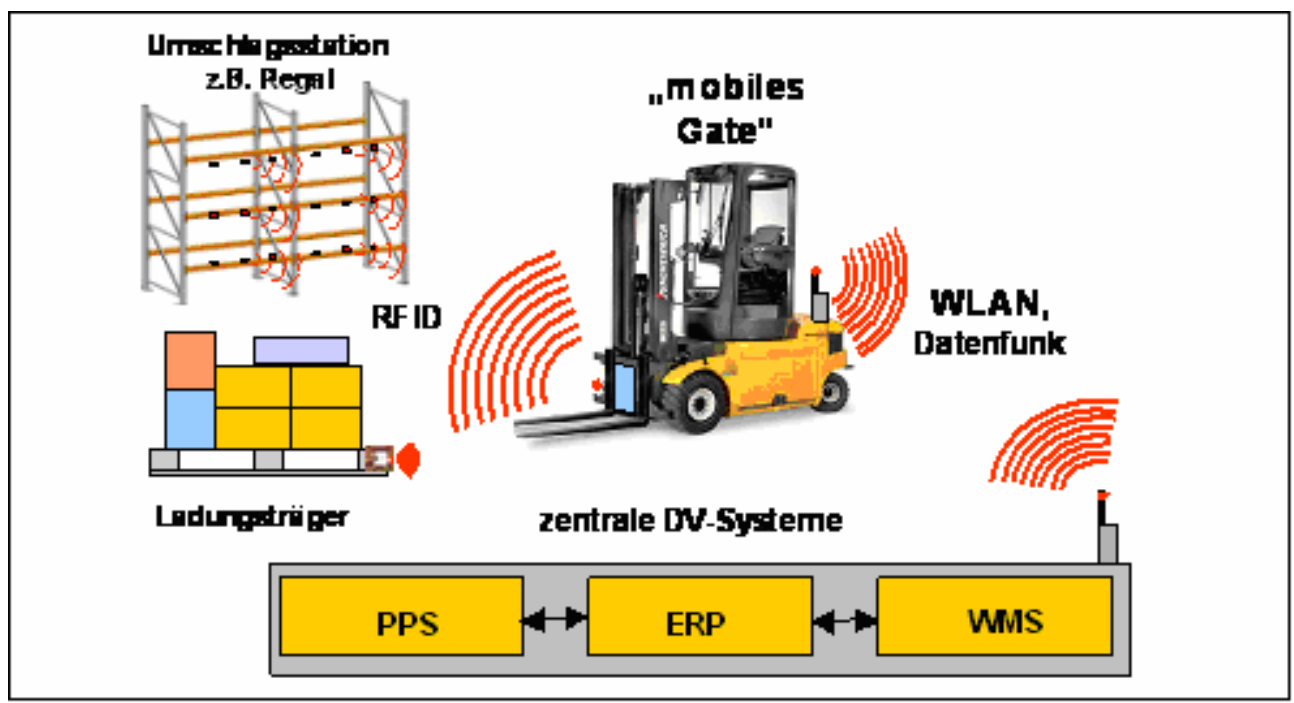

Abbildung: 1

In die Ladungsträger werden RFID-Tags integriert. Sie dienen der Identifikation und können mit Statusinformationen beschrieben werden. RFID-Tags an Lagerort und Arbeitsstation dienen der Materialflussverfolgung während des gesamten Produktionsprozesses und können bei Änderungen im Produktionsablauf dezentral umkonfiguriert werden.

Die Schnittstellen der informationstechnischen, elektronischen und mechanischen Komponenten des Zielführungssystems (RFID-Schreib-/Lesegerät, interaktives Monitoringsystem, WLAN-Schnittstelle) müssen standardisiert werden. Der Informationsaustausch wird über offene Software-Schnittstellen zwischen Zielführungssystem und PPS/ERP/WMS realisiert. Diese Maßnahmen ermöglichen es, alle Abläufe im Materialfluss dezentral zu steuern. Dabei fungiert das Flurförderzeug als mobiles Gate, das im Mittelpunkt des Informationsaustausches steht.'

Bei den Flurförderzeugherstellern gibt es bereits konkrete Vorschläge.

So zeigte Jungheinrich bereits ein Konzept, bei dem Ladungsträger mit Transpondern ausgestattet wurden. Die zusätzliche Ausrüstung von Lagerplätzen mit Transpondern ermöglicht darüber hinaus die einfache Lokalisierung des Lagerbestandes, sowie gestattet Kontrollmöglichkeiten direkt vor bzw. bei Aufnahme des Ladegutes.

Zum Auslesen beider Transponder werden die Flurförderzeuge dazu an geeigneter Stelle mit einer Sende- und Empfangseinheit ausgerüstet. Die empfangenen Daten werden dem Fahrer zunächst auf dem Display angezeigt, können aber auch mittels Datenfunk oder WLAN an übergeordnete Systeme weitergeleitet und dort verarbeitet werden.

Auch wurde bei Jungheinrich über ein System „aktiver Verkehrsschilder“, basierend auf RFID-Technologie nachgedacht. Hierbei wird dem Fahrer bei Annäherung an eine Gefahrenstelle eine Warnung auf dem Display signalisiert. Theoretisch denkbar wäre dabei auch ein Eingriff in die Fahrzeugsteuerung, um die Geschwindigkeit vor Gefahrenpunkten automatisch zu drosseln.

Bei Still ergänzt RFID vorhandene Instrumente wie das Lagerverwaltungs- und Staplerleitsystem (LVS und SLS), die das Unternehmen als Bausteine des Materialfluss-Managementsystems (MMS) anbietet.

Hier wird RFID zur Lokalisierung der Fahrzeuge und zur Optimierung des Betriebsablaufes genutzt. Nach der ersten Erfassung der Ware am Wareneingang erfolgt die Verwaltung der Lagerposition durch eine logische Verknüpfung von Fahrzeugposition und Hubmastsensorik. Diese Position wird in einer Datenbank abgelegt und erlaubt eine Zuordnung von Palette und Ware in einer Datenbank.

Diese wird bei jedem weiteren innerbetrieblichen Transportvorgang der Palette aktualisiert, da das System die Ortsveränderung registriert.

Notwendig hierzu sind Transponder, die regelmäßig in einem Rastermaß von etwa einem Meter im Boden verlegt sind, so dass die Fahrzeugposition immer eindeutig definiert ist. Die etwa 30 mal fünf Millimeter großen Transponder werden in kleinen Bohrlöchern untergebracht, die zum Schutz mit Silikon verschlossen werden. Dies ermöglicht die Installation auch in bereits vorhandenen Lagern und Produktionsstätten. 
Der Kontakt zu den Transpondern wird über eine Antenne im Bodenbereich der Fahrzeuge hergestellt, die die Transponder über ihr Feld auch gleichzeitig mit Energie versorgt.

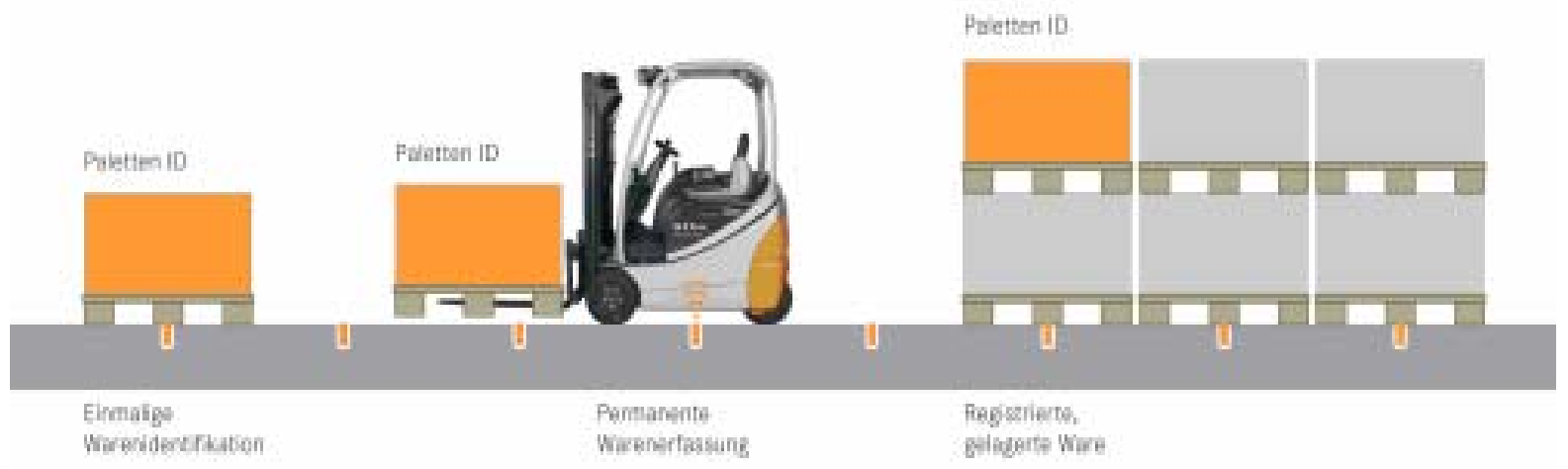

Abbildung 2: Lösung der Firma Still (Quelle: Still)

\section{BMBF-gefördertes Forschungsvorhaben IdentProLog}

Das Projekt IdentProLog dient der Realisierung des zuvor beschriebenen neuen Prozesses. Um eine breite Basis für die Standardisierung von Hard- und Softwareschnittstellen zu schaffen, arbeiten die Jungheinrich AG und die Linde AG/Still GmbH zusammen an diesem Projekt.

Zusammen mit den Forschungsinstituten ITA und IFT, dem Ladungsträgerhersteller Gebhardt, der Firma deister electronic soll der neue Prozess bei der Kuka Roboter GmbH umgesetzt werden. Als Systemintegrator tritt hierbei die Firma PSI Logistics auf.

Es ist geplant, in einem begleitenden Konsortium auch weitere Unternehmen mit einzubeziehen, um auch auf Anwenderseite eine breite Basis zu schaffen. Mit einer an vielfältige Anforderungen angepassten Lösung soll eine große Akzeptanz herbeigeführt werden. Außerdem ist es möglich, dem begleitenden Konsortium auf „informativer Ebene“ anzugehören, ohne sich direkt mit eigenen Ressourcen zu beteiligen. Hierdurch soll der Informationsfluss aus dem Projekt verbessert werden.

Am Anfang steht die Modellierung der Prozesse zur neuen Ablauforganisation. Sie soll Basis für die im Rahmen eines Zielführungssystems umzusetzenden Anforderungen sein. Anschließend wird eine wirtschaftliche Bewertung der neuen Prozesskette durchgeführt. Hiermit soll gezeigt werden, wie hoch die Einsparungen für den Anwender im Endeffekt sein werden. Darüber hinaus liegt der Forschungsschwerpunkt dieser Projektphase in der Definition der Anforderungen an das Zielführungssystem, an die einzusetzende RFID-Technik, an die Ladungsträger und an die Flurförderzeuge. Eine Kundenbefragung soll der Verifikation der definierten Systemanforderungen dienen. Um die Kommunikation der einzelnen Teilsysteme miteinander sicherzustellen, muss zudem ein ebenenübergreifendes Kommunikationskonzept erstellt werden. Hierfür sind insbesondere die Schnittstellen zu den über- und untergeordneten Systemen (z. B. Fahrzeugterminal) zu definieren.

Ein neues, flexibles Zielführungssystem ergibt sich auf Basis der durch die RFID-Komponenten (z. B. an den Ladeeinheiten oder den Lagerplätzen) optimierten Geschäftsprozesse. Auch die Materialübergabepunkte im Produktionssystem (z. B. Bearbeitungszentrum, LKW) sind in dieser Entwicklungsphase informationstechnisch zu gestalten und hinsichtlich der Steuerung des Materialflusses über das Zielführungssystem zu berücksichtigen. Das Zielführungssystem hat die Aufgabe, einen automatisierten Informationsfluss über alle Ebenen sicherzustellen und zugleich im Sinne einer flexiblen Zielführung den physischen Materialfluss zu steuern und optimieren. Im Rahmen des Projektes sollen Stapler mit der RFID-Technik ausgerüstet werden und im Rahmen einer Testphase eingesetzt werden. Die Erprobungsmaßnahmen laufen am Anfang in der Versuchshalle des IFT, zu einem späteren Zeitpunkt erfolgt dann die Umsetzung beim Anwender Kuka Roboter GmbH.

In Abhängigkeit von den Flurförderzeugkomponenten wie bspw. der Gabel ist die flexible Integration von Schreib-/Leseeinheiten, Monitoringsystem/Terminals für den Flurförderzeugfahrer und Kommunikationsschnittstellen mit der Fahrzeugsteuerung in das Flurförderzeug notwendig.Im Bereich der Ladungsträgerhersteller ergibt sich die Aufgabenstellung, dass die Anwender oftmals spezielle Lösungen einsetzen. 
Für die Akzeptanz einer standardisierten Lösung sollen daher unterschiedliche Ladungsträgertypen (Holz, Kunststoff, Stahl) berücksichtigt und die dafür optimale Position der Transponder ermittelt werden.

Die im Projekt zum Einsatz kommenden RFID-Technologien unterliegen der internationalen Standardisierung (ISO 15693, ISO 18000-3, ISO 18000-6, EPCglobal). Diese vorhandenen Standards sollen konsequent angewendet werden. Eine Mitarbeit an diesen Normen ist im Rahmen des Projektes geplant, falls die bestehenden Standards erweitert werden müssen.

Projektbegleitend wird in Abstimmung mit den beteiligten Interessengruppen (Anwender, Anbieter, Nutzer) dafür gesorgt, durch konsistente Implementierungsprofile weitergehende Standards zu entwickeln. Im Zusammenschluss der Flurförderzeug-Hersteller soll die Anwendung der RFID-Technik im Rahmen des Zielführungssystems standardisiert werden. Die Partner streben dabei die Durchsetzung von nationalen und internationalen Normen an.

\section{Ausblick}

Der in Zukunft vollständig transparente innerbetriebliche Materialfluss wird es erstmals zulassen, Optimierungsstragien in Echtzeit zuzulassen. Kleinskalige Logistiknetzwerke werden es in Zukunft gestatten, eine atmende Peripherie aufzubauen, die sich dem Produktionsprozess flexibel anpasst. Transponder werden zukünftig Daten nicht nur speichern, sondern diese auch verarbeiten können um bestimmte Entscheidungen im Materialfluss selbst zu treffen. Dies erlaubt einer Lieferung, sich ihren Weg selbst zu suchen.

\section{Literatur}

[Baginski2006a]

[Baginski2006b]

[Finkenzeller2002]

[Overmeyer2004a]

[Overmeyer2004b]

[Wehking2004]
Baginski, R.: Abschnitt Dezentrale Informationstechnik für Flurförderzeuge und Ladungsträger zur Optimierung der Intralogistik In: Arnold, D (Hrsg.).; Intralogistik. Berlin: Springer, 2006

Baginski, R.: „Finden statt suchen“ - Dezentrale Informationstechnik für Flurförderzeuge und Ladungsträger zur Optimierung der Intralogistik, Tagungsband zu den 8. Karlsruher Arbeitsgesprächen, 2006, S. 92-100

Finkenzeller, K.: RFID-Handbuch : Grundlagen und praktische Anwendungen induktiver Funkanlagen, Transponder und kontaktloser Chipkarten, 3., aktual. Auflage. München: Hanser, 2002.

Overmeyer, L.; Höhn, R.: Logistikdatenerfassung und -verarbeitung in der Produktion auf Basis von intelligenten Transpondern, dhf - Förder-, Lager- und Transporttechnik, Logistik, Automation, November 2004, S. 56-59.

Overmeyer, L.; Vogeler, S.: RFID - Grundlagen und Potenziale, 1. Hannover Kolloquium (Tagungsband), 4./5. November 2004, S. 139-154.

Wehking, K.-H.: Neue Materialflusskomponenten für die zukünftige Logistik, Institut für Fördertechnik und Logistik (IFT) der Universität Stuttgart, dhf-Magazine, Juni 2004, (2004). 